\title{
Die Sushi-Strategie: In 15 Minuten soviel wissen wie in einer Stunde
}

\section{UX-Design eines neuartigen eLearning-Formats für den deutschen Pflegemarkt}

\author{
Dipl.-Des. Oliver Gerstheimer \\ chilli mind $\mathrm{GmbH}$ \\ Königstor 23 \\ 34117 Kassel \\ gerstheimer@chilli-mind.com
}

\section{Cord Krüger}

Bibliomed Medizinische Verlagsgesellschaft mbH

Stadtwaldpark 10

34212 Melsungen

cord.krueger@bibliomed.de

\author{
Dipl.-Des. Sarah Henß \\ chilli mind $\mathrm{GmbH}$ \\ Königstor 23 \\ 34117 Kassel \\ henss@chilli-mind.com
}

\begin{abstract}
Was steckt eigentlich in eLearning oder Blended Learning?

Arbeitgeber, die das Thema Informations- oder Weiterbildungsverantwortung „outsourcen“ und nur daran interessiert sind, dass jemand ein Häkchen setzt und somit bestätigt, dass er Inhalte gesehen hat, wie z. B. Compliance oder Hygienestandards? Oder: Echte und ernsthafte, effiziente wie effektive, aber auch lerndidaktische Aufbereitungen von relevanten Inhalten, die kostenbewusst und skalierbar sind?

Wird im eLearning sorgfältig mit der „Lese- und Wahrnehmungszeit“ der Betrachter und Benutzer umgegangen? Was sind die Erfolgsparameter und Qualitätsvorgaben für eine hohe Akzeptanz und Nutzung? Wird medial mit Gamification-Elementen übersteuert oder in der Dichte und Länge des Content Designs untersteuert?

Vorgestellt wird die UXD-Entwicklung eines eLearning-Formats, also das umfassende Design der prozessualen, wie didaktischen und gestalterischen Regeln und Rahmenvorgaben, nach denen 150 eLearning-Units für den Markt der Pflegenden in Krankenhäusern umgesetzt werden.
\end{abstract}

\section{Keywords}

eLearning, E-Learning, Blended Learning, Formatentwicklung, Lesezeit, Content Design, Lerndidaktik 


\section{Prolog}

\section{Warum eLearning eine gesamtheitliche Format-Konzeption aus Benutzersicht benötigt und nicht nur die fachliche Umsetzung von Einzeleinheiten.}

Jeden Tag passiert in Kliniken der Spagat zwischen einer angemessenen Versorgung der Patienten und einer zeiteffizienten, qualitätsgesicherten und wirtschaftlichen Organisation des Arbeitsalltags. Die Schulung der Mitarbeiter gerät dabei schnell in den Hintergrund und verkommt zur rein rechtlichen Formalität oder zur geforderten Pflichtveranstaltung und Unterweisung, die man „hinter sich bringen möchte und die im Alltag stören. “ Grund für die Demotivation und die Nicht-Akzeptanz der Mitarbeiter sind einerseits die schlechte, heterogene Aufbereitungsqualität der Lerneinheiten, die übersteuerte Anzahl und Zeitlängen von Units, die mit dem realen Arbeitskontext nicht vereinbar sind und schlussendlich beim Benutzer als Zusatzbelastung im Alltag wahrgenommen werden und nicht als Fortbildungschance.

Bei ständig steigenden Anforderungen an Mitarbeiter und Klinikleitung ist hier eine flexible und effiziente Lösung gefragt, die sich an die Ansprüche des jeweiligen Instituts anpasst. Diese Lösung ist Teil des neuen Produktbereichs für ein Verlagshaus mit 50-jähriger Tradition. Durch das Angebot von Fortbildungen für das Krankenhaus wird die Expertise und inhaltliche Qualität des Verlages in einen neuen digitalen Produktbereich verlängert. Um den Wandel vom Zeitschriftenverkäufer zum umfassenden Service-Anbieter erfolgreich zu vollziehen, müssen ein neues Produkt- und Serviceportfolio aufgebaut und neue mediale Prozesse und Expertise etabliert werden.

\section{Vorgehen im Projekt}

Das vorgestellte Projekt erstreckt sich über drei Phasen: die Konzeption und Entwicklung der Lerneinheit und der relevanten Prozesse sowie die Entwicklung eines Corporate Media Designs für das neue Lernformat mit festgelegten Standards in Qualität und passender Kontextualität zum Arbeitsalltag.

\section{Projektphase A: Analyse und Konzeption einer generischen Lerneinheit}

Ausgangspunkt waren die umfassenden Anforderungen aus Stakeholdersicht an ein Fortbildungskonzept aus dem Krankenhausalltag. Sie erfordern eine Lösung, die flexibel in die bestehende Krankenhaus-Systemlandschaft integrierbar ist, die aktuelle und geprüfte Inhalte enthält und eine einfache Bedienung sowie effizientes Lernen ermöglicht. Weiter wurden aktuelle Entwicklungen auf dem Weiterbildungsmarkt Richtung kompakten und komprimierten Formaten, integrierten Lernkonzepten und benutzergerechten Lernen einbezogen. Eine umfassende Marktanalyse mit 10 Fokusgruppen und Experten wurden zur Validierung der Anforderungen durchgeführt. Ziel war die Konzeption einer exemplarischen 
Lerneinheit. Anhand von zwei Prototypen wurden die Dichte der Fakten, die Lesezeit über die gesamte Unit, das generische Framework und erste Gestaltungselemente getestet.

\section{Projektphase B: Entwurf und Gestaltung einer Product Identity für Marke und Lerninhalte}

Ziel dieser Phase war die Entwicklung einer Product Identity der neuen Formate des eLearning Angebots durch die Definition von Anforderungen und die Gestaltung relevanter Elemente. Parallel zur Entwurfsphase wurde die definierte Gestaltung anhand eines ersten Moduls mit zwei Lerneinheiten prototypisch getestet. Die Prototypen wurden mit den finalen Umsetzungsprogrammen (Adobe Captivate und Storyline) erstellt.

\section{Das Ergebnis}

Die Formatentwicklung umfasst dabei den gesamtheitlichen Prozess vom inhaltlichen Portfoliomanagement über das Angebot der Units bis zum Content Design, die mediale Content-Aufbereitung im Spannungsfeld von Fachexperten und Umsetzern sowie das Konzept einer generischen Lerneinheit, die den Ansprüchen des Krankenhausalltags gerecht wird.

\section{Ein gesamtheitlicher Prozess}

Damit eine Lerneinheit entsteht, durchläuft sie einen dreistufigen Prozess. Dieser Prozess wurde im Projekt mitentwickelt, um eine standardisierte Erstellung der Lerneinheiten sicherzustellen. Die drei Stufen sind:

1. Die strategische Themenauswahl

2. Die Erstellung des Contents

3. Die Produktion der Formate und Lerneinheiten

Die Prozessphasen haben jeweils unterschiedliche Ziele, Dokumente und Beteiligte. Jede Lerneinheit des Portfolios durchläuft diesen standardisierten, Prozess um eine konstante Qualität über das gesamte Portfolio sicherzustellen.

\section{Prozessphase 01: Strategische Themenauswahl}

Fokus dieser Prozessphase ist es, im Rahmen eines aktiven Portfoliomanagements aus einer Sammlung von möglichen Themen die für die nächste Umsetzungsphase relevanten auszuwählen, so dass ein stimmiges und attraktives Gesamtportfolio für das Krankenhaus entsteht. Zur Durchführung dieser Prozessphase wurde als Masterdokument eine ExcelDatei, das Themenportfolio, erstellt. Der Prozess der strategischen Themenauswahl erfolgt in mehreren Stufen: Von der Recherche und Sammlung relevanter Themen für das Krankenhaus über den Diskurs im Fachbeirat bis hin zur Vorbereitung ausgewählter Themen für die Content-Erstellung. 


\section{Prozessphase 02: Content-Erstellung}

Ziel ist es, den Content für die Lerneinheit in einem iterativen Prozess zu optimieren und in Medienformaten umzusetzen. Zuerst findet ein Einsammeln und Verdichten des Wissens der Lerneinheit statt. Der Fokus dieser Phase liegt auf der effizienten Einholung, Verdichtung und Prüfung des Wissens. Anschließend wir die Mediale Umsetzung geplant. Der Fokus dieser Phase liegt auf der Auswahl eines sinnvollen Mediums (Foto, Animation, Realfilm) sowie der Vorbereitung zur Produktion. Das Masterdokument dieser Phase durchläuft hierbei drei Entwicklungsstufen vom Experten-Wizard, über das Fakten-Storyboard zum MediaStoryboard.

\section{Prozessphase 03: Format-Produktion}

Ziel dieser Prozessphase ist es, durch die Produktion, Iteration und Abnahme von Prototypen, die Elemente der Lerneinheiten in einem seriellen, planbaren und skalierbaren Prozess herzustellen. Der Fokus liegt dabei auf einer standardisierten und durchgängigen Qualität. Die Themen werden auf Basis des Media-Storyboards sowie der Spezifikation und der Media Guidelines umgesetzt.

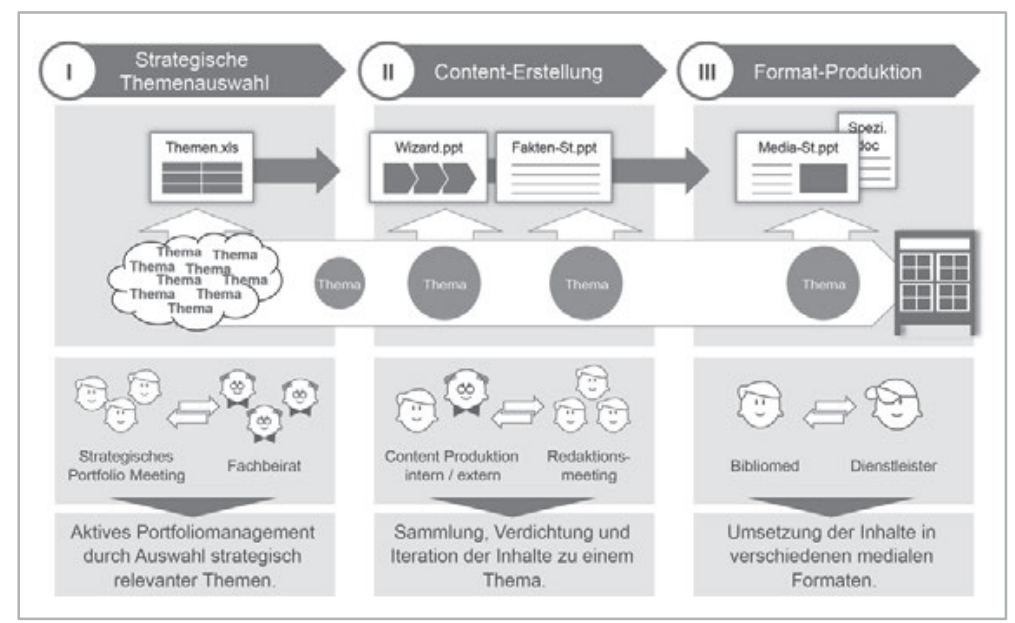

Abbildung 1: Gesamtprozess zur Erstellung einer Lerneinheit

\section{Formatentwicklung einer Lerneinheit}

In der Lerneinheit wird über einen fest definierten Zeitraum von 15 Minuten Wissen in Form von Fakten zu einem bestimmten Thema vermittelt. Die Herausforderung besteht in der effektiven und effizienten Vermittlung von Inhalten und Wissen, die mit Erlebnisattraktivität über Onlinekanäle aufbereitet und mit neuen und innovativen Lern-Produkten und -Services konzeptioniert und umgesetzt wird. Folgende Merkmale wurden hierfür angedacht: 
Der didaktische Aufbau der Lerneinheiten ist gekennzeichnet von einem generischen, wiederkehrenden Framework. Jede Lerneinheit beginnt und endet mit den gleichen Elementen, hat die gleiche Länge und eine generische Struktur der Inhalte. Der Benutzer kann im gewohnten Raster lernen und findet schnell Einstieg in neue Themen. Die Wissensvermittlung wird durch Schätzfragen unterbrochen, die den Benutzer aktivieren. Die Fakten einer Lerneinheit werden nach Wissen eines bestimmten Typs durchsucht. Das Wissen in Kategorien wie Praxis, Recht uns Selbstschutz wird in der Lerneinheit hervorgehoben. Beispielsweise werden Informationen hervorgehoben, die die Krankenschwester bei der Durchführung eines Arbeitsschritts schützen, z. B. das Anlegen von Handschuhen bei einer Untersuchung.

Die Fakten werden in der zweiten Phase der Content-Erstellung im Media Storyboard von Textfakten in ein sinnvolles Medienformat übersetzt. So wird sichergestellt, dass die unterschiedlichen Inhalte optimal vermittelt werden.

Die Qualitätssicherung der Inhalte über mehrere Stufen ist fest im Prozess der Erstellung einer Lerneinheit verankert: relevante Themen werden vom Fachbeirat festgelegt, das Wissen wird von Experten aus der Praxis erstellt, die Inhalte werden über Redaktion und Zweitexperten validiert und final abgenommen.

Mehrere Lerneinheiten bauen aufeinander auf, bilden einen Lernpfad und können mit einer Zertifizierung enden. Hierdurch kann der Benutzer sein Wissen stufenweise vertiefen, die Personalabteilung Wissensstufen für Positionen definieren und über das LernManagement-System Gruppen zuweisen. Diese Zertifizierung wird in einem Test erworben. Sie kann ein offizielles Dokument zur Bezeugung einer Qualifikation sein. Vorausgesetzt die Lerneinheit wurde durch ein Institut wie die „Pflegekammer“ zertifiziert.

\section{Fazit}

Durch den gestalteten Prozess sowie das vermittelte Format entsteht in kurzer Zeit eine hohe didaktische Lernqualität der Inhalte. Die neuen Formate und Medien werden Mitarbeitern oder Fachbenutzern über ein Lern-Management-System verfügbar gemacht. Die Zukunft der Weiterbildung und des Wissenstransfers in Unternehmen und Institutionen entwickelt sich radikal in Richtung Blended Learning. Das Lernangebot ist so konzipiert, dass es zukünftig durch Präsenzveranstaltungen vor Ort im Krankenhaus ergänzt werden kann. So lassen sich theoretische Inhalte im eLearning vermitteln, die anschließend in einer kurzen Präsenzveranstaltung mit den gleichen Experten angewandt und wiederholt werden.

Die Ausgestaltung passgenauer und qualitativ hochwertiger eLearning-Formate mit standardisierten Qualitätsparametern ist Trumpf. Das bestehende Angebot für Kliniken bewegt sich zwischen Großanbietern mit einem riesigen Themenangebot und kleinen Einzelanbietern, spezialisiert auf bestimmte Bereiche mit einer geringen Anzahl an Einheiten. Die Lerneinheiten ähneln theoretischen Veröffentlichungen und werden vornehmlich aus Printmedien kompiliert oder haben keine geeignete Lösung für eine technische Anbindung. 
Lese- und Interaktionszeit, der didaktische Anspruch, aber auch die „Schere“ zwischen den Produktionskosten einer eLearning-Unit bilden dabei den Anforderungsrahmen. Ein standardisiertes Vorgehen im Erstellungsprozess sowie die Auswahl effizienter Umsetzungstools machen Prozess und Umsetzung effizient. Die geplante Lese- und Interaktionszeit wird bereits bei der Erstellung der Inhalte im Wizard bedacht und in Form von Fakten- und Zeichenanzahl vorgegeben. Kurz auf den Punkt: Es geht nicht um irgendein „stumpfes oder übermediatisiertes Angebotsportfolio“ von langweiligen Wissensinhalten, sondern um die echte UX-Gestaltung eines spaßvollen eLearning-Formats, welches die Bedürfnisse der Benutzer und Kunden nach effizientem Lernen und Wissen exakt adressiert und zum kontextuellen Arbeitsalltag passt.

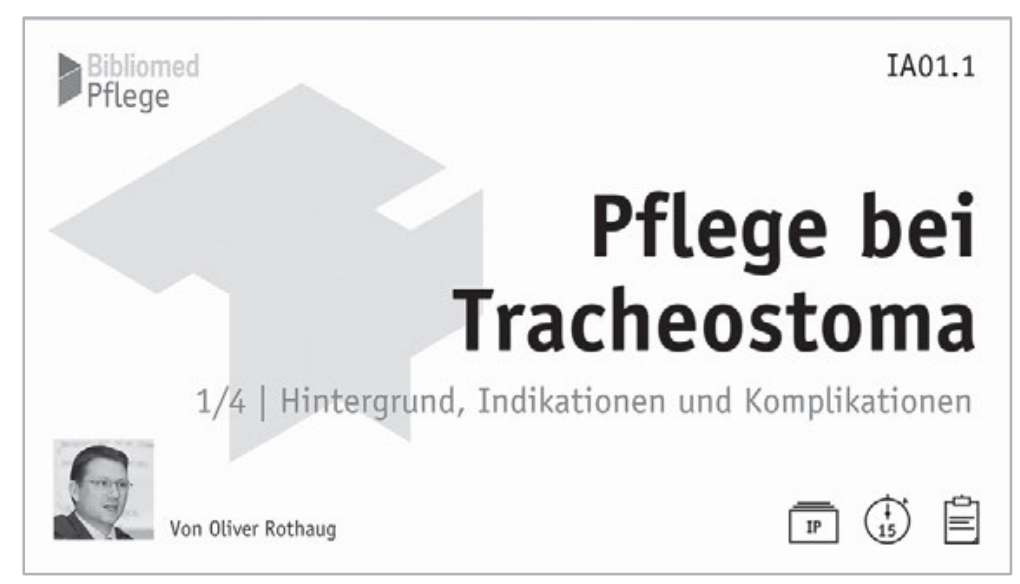

Abbildung 2: Titeldesign einer Lerneinheit

\section{Helden im Projekt}

\section{Helden der Informationsarchitektur:}

- Standardisierung der Laufzeit einer Lerneinheit (15 Min.). Die Anzahl der vermittelbaren Inhaltsfakten beträgt ca. 75-100 Fakten (z. B. Bullet Points),

- Fokus auf eine adäquate Komprimierung und Faktenstaffelung durch grafische Unterstützungen zur schnelleren Wahrnehmung,

- Planung von Inhalten zur Mehrfachverwertung über die Lerneinheit hinaus als „Best of“ von Querschnittsthemen wie rechtliche Grundlagen in der Pflegepraxis,

- Entwickeln einer generischen Struktur der 15-minütigen Lerneinheit mit sich wiederholenden Rahmenelementen, 


\section{Helden der Systematik und des Aufbaus:}

- Konfektionierung der einzelnen Lerneinheiten in Lernpfaden und Abschluss eines Themenpakets über finale Abschlusstests unter Prüfungsbedingungen,

- Erwerb von Zertifikaten oder Punkten bei relevanten Instituten der Pflegeberufe;

- Indexierung der Lerneinheiten über das gesamte Portfolioregal zur Optimierung der „Findability“ in digitalen Verortungen,

\section{Helden im Prozess:}

- strategische Auswahl der Themen unter Einbeziehung eines Expertenbeirats;

- zeit-, budget-effiziente wie planbare Content-Einholung und Iteration,

- gezielte Weiterverarbeitung und Komprimierung der Inhalte über das Fakten-Storyboard sowie Auswahl passender medialer Lern- und Umsetzungsmuster,

- Planung, Kalkulation und Iteration der medialen Umsetzung über das Media-Storyboard als Übergabedokument an verschiedene Dienstleister,

- regelbasierte Qualitäts-Standardisierung von Konzept, Prozess und Design über Spezifikation und Media-Design-Guidelines 